\title{
TRPV4-pathy, a novel channelopathy affecting diverse systems
}

\author{
Jin Dai ${ }^{1,2}$, Tae-Joon Cho ${ }^{3}$, Sheila Unger ${ }^{4}$, Ekkehart Lausch $^{4}$, Gen Nishimura ${ }^{5}$, Ok-Hwa Kim ${ }^{6}$, \\ Andrea Superti-Furga ${ }^{4}$ and Shiro Ikegawa ${ }^{1}$
}

Transient receptor potential cation channel, subfamily V, member 4 (TRPV4) is a calcium-permeable nonselective cation channel of unknown biological function. TRPV4 mutation was first identified in brachyolmia, and then in a spectrum of autosomaldominant skeletal dysplasias, which includes Kozlowski type of spondylometaphyseal dysplasia, metatropic dysplasia, Maroteaux type of spondyloepiphyseal dysplasia and parastremmatic dysplasia. Recently, TRPV4 mutation has also been identified in a spectrum of neuromuscular diseases that includes congenital distal spinal muscular atrophy (SMA), scapuloperoneal SMA, and hereditary motor and sensory neuropathy type IIC. These diverse spectrums of diseases compose a novel channelopathy, TRPV4-pathy, which could further include polygenic traits such as serum sodium concentration and a chronic obstructive pulmonary disease. In this review, we clarified the TRPV4 mutation spectrum, and discussed the phenotypic complexity of TRPV4-pathy and its pathogenic mechanisms. TRPV4-pathy may extend further to other monogenic and polygenic diseases. Journal of Human Genetics (2010) 55, 400-402; doi:10.1038/jhg.2010.37; published online 27 May 2010

Keywords: mutation; neuropathy; skeletal dysplasia; TRPV4; TRPV4-pathy

\section{INTRODUCTION}

Three interesting articles have recently been published head to tail in Nature Genetics, ${ }^{1-3}$ which report identification of mutations in the gene encoding the transient receptor potential cation channel, subfamily V, member 4 (TRPV) ${ }^{4}$ in a spectrum of neuromuscular diseases, which includes congenital distal spinal muscular atrophies (SMAs, also known as hereditary motor neuropathies), scapuloperoneal SMA, and hereditary motor and sensory neuropathy type IIC (HMSN IIC, also known as HMSN2C or Charcot-Marie-Tooth disease type $2 \mathrm{C}$ ). These diseases are all disorders of the peripheral nervous system transmitted as autosomal-dominant traits, but are clinically very heterogeneous. Thus, the discovery that they share a common genetic origin came as a surprise.

TRPV4 is a calcium-permeable nonselective cation channel of unknown biological function. ${ }^{4}$ Mutations in TRPV4 had originally been found in a different category of disorders; autosomal-dominant skeletal dysplasias that includes brachyolmia, spondylometaphyseal dysplasia, Kozlowski type and metatropic dysplasia. ${ }^{5-7}$ Recently, we have found TRPV4 mutations in two additional skeletal dysplasias, spondyloepiphyseal dysplasia, type Maroteaux (or pseudo-Morquio, type 2 ) and parastremmatic dysplasia. ${ }^{8}$ Altogether, 29 different mutations have been found in the 'family' of skeletal dysplasias composed of these overlapping, but distinctive entities ${ }^{5-8}$ (Figure 1).
The pathogenic mechanisms entailed by these TRPV4 mutations remain unclear, and the new observation of the 'neuromuscular' phenotypes by the Nature Genetics papers ${ }^{1-3}$ makes it all the more interesting. Now, we are shown that the two different groups of diseases-one is the progressive degenerative disorder of the peripheral nerves and the other is the failure of formation and development of hard skeletal tissues manifesting very early in life-are allelic. The observations that a TRPV4 polymorphism (P19S) is significantly associated with serum sodium concentration and with hyponatremia, ${ }^{9}$ and TRPV4 SNPs are significantly associated with a chronic obstructive pulmonary disease ${ }^{10}$ further underline the extraordinary pleiotropism of TRPV4 mutations. It is noteworthy that neither the two groups of monogenic diseases nor the polygenic disorders show essential phenotypic overlap among each other.

In the face of this phenotypic complexity of the TRPV4-related disease (TRPV4-pathy), we suspect that the pathogenic explanation proposed by these papers could be too simplistic. Four missense mutations at three distinct positions in the TRPV4 protein have been identified in the neuromuscular disorders, all four mutations are within the ankyrin (ANK) repeats of TRPV4, and all involve substitutions of arginine residues. ${ }^{1-3}$ Although most 'skeletal' TRPV4 mutations are also missense mutations, they also include a single amino-acid deletion ${ }^{7}$ and a frame-shift mutation ${ }^{8}$ (Figure 1). The clustering of 'neuropathic' mutations in the ANK domain is

${ }^{1}$ Laboratory for Bone and Joint Diseases, Center for Genomic Medicine, RIKEN, Tokyo, Japan; ${ }^{2}$ The Center of Diagnosis and Treatment for Joint Disease, Drum Tower Hospital Affiliated to Medical School of Nanjing University, Nanjing, China; ${ }^{3}$ Department of Orthopaedic Surgery, Seoul National University Children's Hospital, Seoul, Korea; ${ }^{4}$ Centre for Pediatrics and Adolescent Medicine, University of Freiburg, Freiburg, Germany; ${ }^{5}$ Department of Radiology, Tokyo Metropolitan Kiyose Children's Hospital, Kiyose, Japan and ${ }^{6}$ Department of Radiology, Ajou University Hospital, Suwon, Korea

Correspondence: Dr S Ikegawa, Laboratory of Bone and Joint diseases, Center for Genomic Medicine, RIKEN, 4-6-1, Shirokanedai, Minato-ku, Tokyo 108-8639, Japan. E-mail: sikegawa@ims.u-tokyo.ac.jp

Received 26 February 2010; revised 30 March 2010; accepted 2 April 2010; published online 27 May 2010 


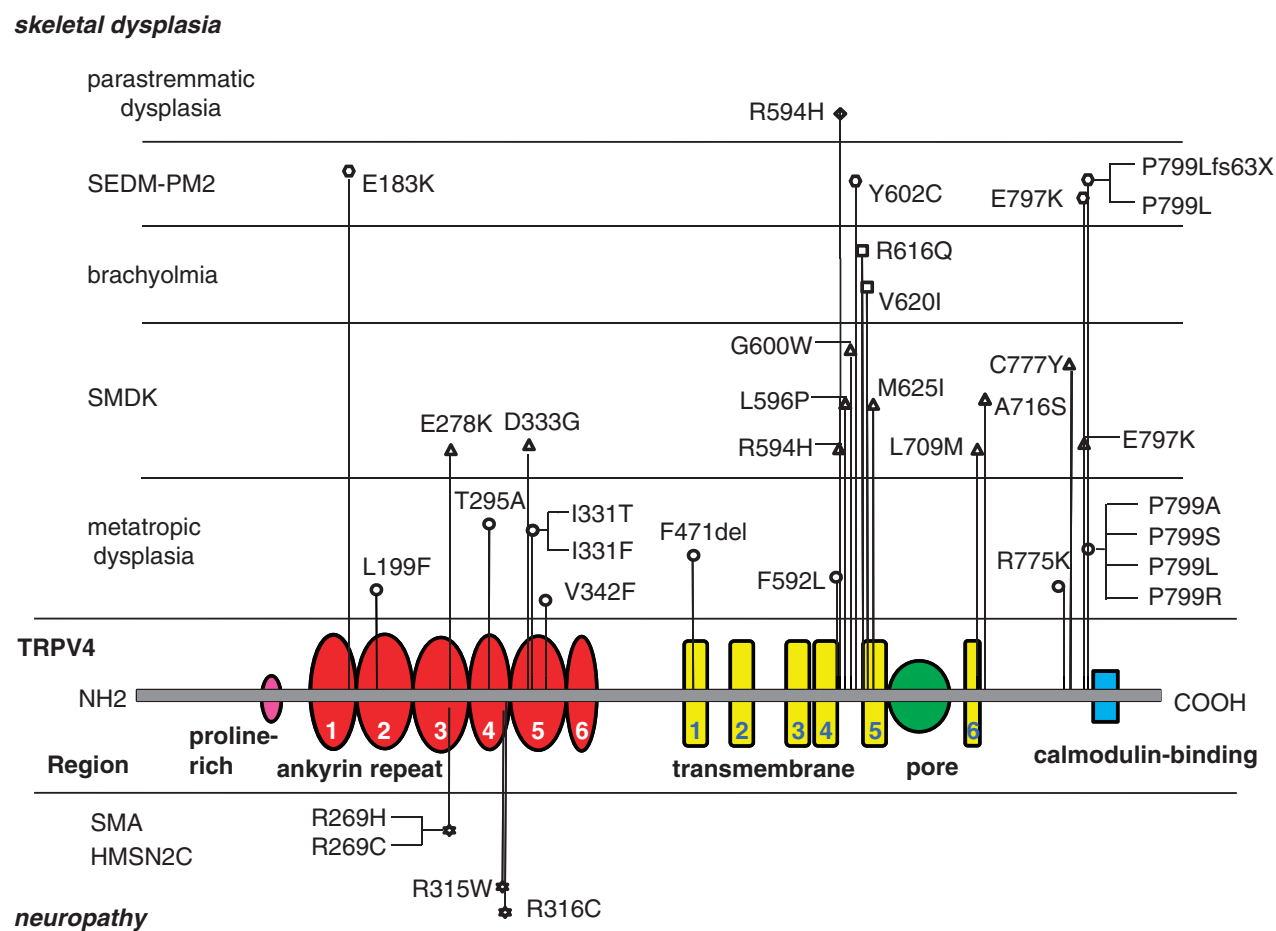

Figure 1 TRPV4 mutations in skeletal dysplasias and neuropathies. HMSN2C, hereditary motor and sensory neuropathy 2C; PM2, pseudo-Morquio, type 2; SEDM, spondyloepiphyseal dysplasia, type Maroteaux; SMA, spinal muscular atrophy; SMDK, spondylometaphyseal dysplasia, type Kozlowski.

emphasized in the Nature Genetics papers, ${ }^{1,3}$ but ANK mutations have been observed in many 'skeletal' phenotypes as well (Figure 1). It seems equally unlikely that substitution of arginine may have a special significance, as $\mathrm{R} 594 \mathrm{H}$ is a very frequent mutation in the skeletal group. ${ }^{6,7}$ Moreover, arginine residues in the ANK domains are not conserved between human TRPV proteins, whereas many of surrounding residues are conserved (Supplementary Figure 1). Interestingly, the residues corresponding to arginine 269 of TRPV4 are histidine in TRPV1 and cysteine in TRPV2 - precisely the substitutions observed by the papers (R269H and R269C). ${ }^{1-3}$ If the arginine 269 were indeed crucial to the neuromuscular phenotypes, the mechanism might be a functional trans-specification of TRPV4 toward TRPV1 or TRPV2.

Functional studies would probably shed light on the consequence of distinct mutations; however, present results and conclusion of functional analyses are diverse among the studies. Auer-Grumbach et al. ${ }^{1}$ found affected channel maturation that could lead to the reduced surface expression of functional TRPV4 channels. Deng et $a .^{2}$ concluded that increased calcium channel activity is a distinct property. Landouré et al. ${ }^{3}$ suggested a loss of normal TRPV4 function as well as the toxic effects of abnormal channel opening and calcium influx. A gain-of-function characterized by increased constitutive activity and elevated channel activation by a variety of mechanisms has been suggested for the skeletal disorders. ${ }^{5,6}$ One of the skeletal mutations predicts abolition of the normal C-terminal from P799 and substitution by a stretch of 63 missense amino acids; ${ }^{8}$ as the C-terminus of TRPV4 is required for transport to the cell membrane, ${ }^{11}$ it seems difficult to hypothesize that the gain-of-function mechanism alone could be responsible. Simple gain and loss of channel activity are also unlikely to explain the two distinct phenotypic groups. As an alternative explanation, mutant TRPV4 proteins might induce aberrant signaling from the endoplasmic reticulum; a similar phenomenon is known to occur with several FGFR3 mutations. $^{12,13}$

At the current state of knowledge, it appears that the phenotypic diversity of TRPV4 mutations cannot be explained by a simple gain/ loss-of-function mechanism, but most likely involve additional molecular partners-for example, proteins interacting with specific domains of TRPV4 or other members of the TRPV4 family that would form heteromultimers with TRPV4-and affect distinct signaling networks. Further accumulation of knowledge on human mutations and detailed evaluation of disease phenotypes would give clue into the pathogenic mechanism. Given the wide range of tissues expressing TRPV4, ${ }^{4}$ we would not be surprised if the spectrum of TRPV4-pathy would extend still further to other monogenic and/or polygenic diseases. In addition, we would not be surprised if other TRPV-pathies would be found.

\section{ACKNOWLEDGEMENTS}

This project is supported by Grants-in-aids from the Ministry of Education, Culture, Sports and Science of Japan (No. 21249024) from Research on Child Health and Development (No. 20-S-3), and from the Ministry of Health, Labor and Welfare of Japan (Measures for Intractable Diseases 046 in 2010).

1 Auer-Grumbach, M., Olschewski, A., Papić, L., Kremer, H., McEntagart, M. E. Uhrig, S. et al. Alterations in the ankyrin domain of TRPV4 cause congenital distal SMA, scapuloperoneal SMA and HMSN2C. Nat. Genet. 42, 160-164 (2010).

2 Deng, H. X., Klein, C. J., Yan, J., Shi, Y., Wu, Y., Fecto, F. et al. Scapuloperoneal spinal muscular atrophy and $\mathrm{CMT} 2 \mathrm{C}$ are allelic disorders caused by alterations in TRPV4. Nat. Genet. 42, 165-169 (2010).

3 Landouré, G., Zdebik, A. A., Martinez, T. L., Burnett, B. G., Stanescu, H. C., Inada, H. et al. Mutations in TRPV4 cause Charcot-Marie-Tooth disease type 2C. Nat. Genet. 42 170-174 (2010). 
4 Everaerts, W., Nilius, B. \& Owsianik, G. The vallinoid transient receptor potential channel Trpv4: from structure to disease. Prog. Biophys. Mol. Biol. (e-pub ahead of print 14 October 2009; doi:10.1016/j.pbiomolbio.2009.10.002).

5 Rock, M. J., Prenen, J., Funari, V. A., Funari, T. L., Merriman, B., Nelson, S. F. et al. Gain-of-function mutations in TRPV4 cause autosomal dominant brachyolmia. Nat. Genet. 40, 999-1003 (2008).

6 Krakow, D., Vriens, J., Camacho, N., Luong, P., Deixler, H., Funari, T. L. et al. Mutations in the gene encoding the calcium-permeable ion channel TRPV4 produce spondylometaphyseal dysplasia, Kozlowski type and metatropic dysplasia. Am. J. Hum. Genet. 84, 307-315 (2009).

7 Dai, J., Kim, O. H., Cho, T. J., Schmidt-Rimpler, M., Tonoki, H., Takikawa, K. et al. Novel and recurrent TRPV4 mutations and their association with distinct phenotypes within the TRPV4 dysplasia family. J. Med. Genet. (in press).

8 Nishimura, G., Dai, J., Lausch, E., Unger, S., Megarbané, A., Kitoh, H. et al. Spondyloepiphyseal dysplasia Maroteaux type (pseudo-Morquio syndrome type 2) and parastremmatic dysplasia are caused by TRPV4 mutations. Am. J. Med. Genet. (in press).
9 Tian, W., Fu, Y., Garcia-Elias, A., Fernández-Fernández, J. M., Vicente, R., Kramer, P. L. et al. A loss-of-function nonsynonymous polymorphism in the osmoregulatory TRPV4 gene is associated with human hyponatremia. Proc. Natl Acad. Sci. USA. 106, 1403414039 (2009).

10 Zhu, G., ICGN Investigators, Gulsvik, A., Bakke, P., Ghatta, S., Anderson, W. et al. Association of TRPV4 gene polymorphisms with chronic obstructive pulmonary disease. Hum. Mol. Genet. 18, 2053-2062 (2009).

11 Becker, D., Müller, M., Leuner, K. \& Jendrach, M. The C-terminal domain of TRPV4 is essential for plasma membrane localization. Mol. Membr. Biol. 25, 139-151 (2008).

12 Lievens, P. M., Roncador, A. \& Liboi, E. K644E/M FGFR3 mutants activate Erk1/2 from the endoplasmic reticulum through FRS2 alpha and PLC gamma-independent pathways. J. Mol. Biol. 357, 783-792 (2006).

13 Lievens, P. M., De Servi, B., Garofalo, S., Lunstrum, G. P., Horton, W. A. \& Liboi, E Transient dimerization and interaction with ERGIC-53 occur in the fibroblast growth factor receptor 3 early secretory pathway. Int. J. Biochem. Cell Biol. 40, 2649-2659 (2008).

Supplementary Information accompanies the paper on Journal of Human Genetics website (http://www.nature.com/jhg) 